
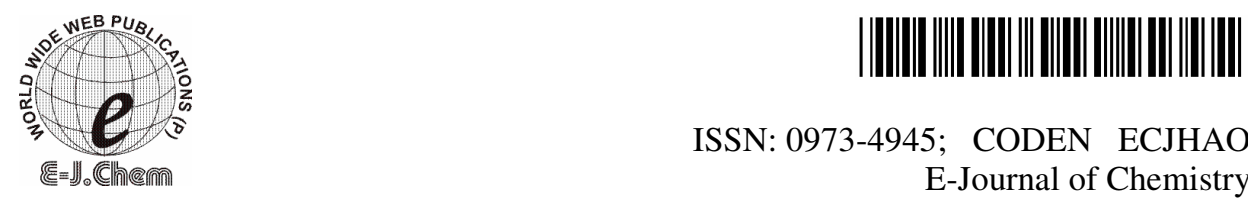

ISSN: 0973-4945; CODEN ECJHAO

E-Journal of Chemistry

http://www.e-journals.net 2009, 6(3), 819-829

\title{
Mechanism of Trees / Bushes Like Pattern in Chemical System
}

\author{
P. K. SRIVASTAVA* and NARENDRA YADAV \\ Department of Applied Chemistry, \\ Birla Institute of Technology, Mesra, Ranchi - 835 215, India. \\ pk_srivastavapk@rediffmail.com
}

Received 7 September 2008; Accepted 30 October 2008

\begin{abstract}
Trees / bushes like pattern have been investigated in succinic acid + ethylacetoacetate $+\mathrm{BrO}_{3}^{-}+\mathrm{Fe}$ (phen) ${ }_{3}^{+2}+\mathrm{Ce}^{+4}+\mathrm{H}_{2} \mathrm{SO}_{4}$ system at $30{ }^{\circ} \mathrm{C}$ in a petridish after 6 hours. These patterns are dependent on initial concentration of reactants and temperature. The system first shows fast appearing / disappearing blue and red pattern then bifurcate into concentric waves and finally shows a tree / bushes type pattern which grows in length and breadth against time. Microphotographs of trees / bushes pattern, succinic acid and ceric ammonium sulphate shows that a new compound has been formed which is responsible for this pattern. It is further confirmed by X-ray powder spectrum of trees / bushes pattern material and succinic acid. IR spectra of pattern material show that presence of cyclic compound in which $\mathrm{Ce}^{+4}$ ion coordinated by hydroxyethylacetoacetate. A tentative mechanism of pattern formation is proposed.
\end{abstract}

Keywords: Trees, bushes like pattern, Mechanism, Coordination, Hydroxyethylacetoacetate

\section{Introduction}

Reactions that show oscillations in open system give rise to a range of interesting wave behaviour in unstirred systems. Clock reactions give rise to one-off reaction-diffusion fronts, with the reactants being completely consumed as the front passes ${ }^{1-2}$. In B-Z-type systems, the front corresponds to a wave of reactions involving the intermediates, with little consumption of major reactants. The system have a wave "break" following the front, returning the systems to its original, pre-front state and gives rise to a pulse. If there are further initiations, the system will also be able to support further waves, possibly giving rise to a series of pulses, a wave train. These wave trains can organize the reaction mixture in two or three dimensions into a variety of intricate evolving patterns ${ }^{3-5}$. Many of these studies of waves in the B-Z system have been carried out in thin films of solutions in petridish. The simplest situation arises when the reaction mixture is excitable, rather than spontaneously 
oscillatory $^{6}$. A point source initiation then naturally gives rise to an expanding circular wave pulse. Once these have expanded sufficiently, they propagate much as one-dimensional wave's. In the presence of a periodic or continuous stimulations, a series of concentric rings, giving rise to a target patterns of blue circles nested on red background, emerges. The inner rings rely on the resetting of the intermediate concentration and so are not possible in oneoff clock type reactions. The spontaneous initiation of the waves in a target pattern appears to rely on the presence of heterogeneities, such as the presence of dust-particles or defects in the surface of the petridish. Careful filtering suppresses this spontaneous initiation in excitable systems. Each target reflects the frequency of the initiation at its center. If this is caused by random heterogeneities, different target cores may naturally have different frequencies. The speed of the waves varies from one target to another, with low frequencies leading to higher velocities. These two effects mean that the waves are more closely spaced for high frequency sources, i.e. these have a shorter wavelength. When waves from two targets colloid, they annihilate each other. As the subsequent wave for the higher frequency patterns comes closer on the heels of the annihilated wave than that for the low frequency source, the next collision occurs closer to the later. Over the course of several collisions, waves from the higher frequency source eventually reach the vicinity of the low frequency core, which is then also annihilated. Higher frequency targets grow at the expense of low frequency ones; given sufficient time, the higher frequency source will entertain the whole dish. For a given target pattern also reveals that the outer most wave travels at a slightly higher speed than subsequent, inner waves, all of which have essentially the same speed.

Far from, equilibrium growth phenomena ${ }^{7-9}$ has been reported in electrochemical deposition. Irreversible aggregation of small particles to form large clusters is technologically and scientifically important ${ }^{10-12}$. The formation of patterns and shapes of chemical and biological systems have considerable interest. The study of morphological stability of growing bodies, crystal growth, development of chemical waves and rhythmic crystallization in gel media offers one possibility for investigating non-equilibrium structures in physicochemical and biological systems ${ }^{13-14}$. Euclidean geometry have been used to model man-made objects ${ }^{15}$ and fractal geometry to model natural objects in computer. ${ }^{16-17}$ Bushes and tree like patterns in B-Z system seems to be interesting for elucidating the mechanism of natural patterns in biological systems.

\section{Experimental}

Succinic acid, ethylacetoacetate, $\mathrm{H}_{2} \mathrm{SO}_{4}(\mathrm{CDH}), \mathrm{FeSO}_{4} \quad 7 \mathrm{H}_{2} \mathrm{O}$ (S.D. Fine) 1-10phenonphthroline $(\mathrm{CDH})$, ammonium ceric sulphate and $\mathrm{KBrO}_{3}$ (E.Merck) of analytical grade were used as such without further purification. Experiment was performed in a completely flat petridish of $9.1 \mathrm{~cm}$ inner diameter. The experimental table was completely labeled by sprit level. The movement of the front and growth of the trees and bushes like pattern were measured by cathodometer. The photographs of patterns were taken by a zoom lens camera. The pattern material was completely soluble in methanol, and was purified by recrystallizing in methanol. The microphotographs of the crystallized pattern material were taken by Carlzeiss's ZENNAMED-2 electronic microscope with photographic attachment at the magnification of 400X. The IR spectrum was taken by an ABB Bomen FTLA 2000 FTIR. X-ray spectrum was taken by Rigaku X-ray spectrophotometer.

\section{Results and Discussion}

Ethylacetoacetate $=[0.1212 \mathrm{M}]$, Succinic Acid $=[0.1016 \mathrm{M}]$, Ceric ammonium sulphate $=$ $[0.00486 \mathrm{M}], \mathrm{Fe}(\mathrm{Phen})_{3}{ }^{+2}=[0.0038 \mathrm{M}], \mathrm{H}_{2} \mathrm{SO}_{4}=[0.42 \mathrm{M}]$ was taken in a petridish $9.1 \mathrm{~cm}($ i.d. $)$ 
and thickness of the solution was kept $0.1 \mathrm{~cm}$. The reaction was started by adding $\mathrm{BrO}_{3}=$ $[0.0553 \mathrm{M}]$ and making it homogeneous. Immediately after addition of $\mathrm{BrO}_{3}$ red color of solution turns into blue color and after a break of a minute it again turns into red color. Then color of the solution changes periodically from blue to red for 5 to 8 cycle within two to three minutes with decreasing frequency. A few stages of the color change are shown in Figure 1.

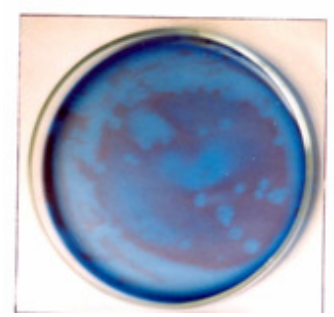

a

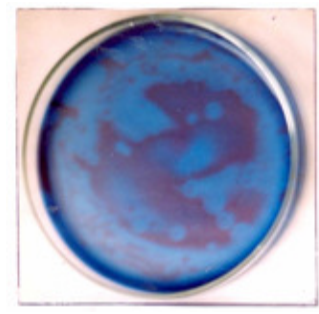

b

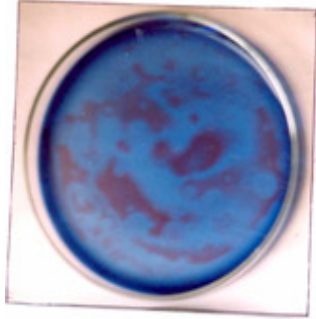

$\mathrm{c}$

Figure. 1. Fast appearing pattern up to 3 minutes.

Composition: Ethylacetoacetate [0.1212 M], Succinic Acid, [0.1125 M], Ce ${ }^{+4}[0.00486 \mathrm{M}]$, $\mathrm{Fe}$ (phen) ${ }_{3}{ }^{+2}[0.0038 \mathrm{M}], \mathrm{H}_{2} \mathrm{SO}_{4}[0.42 \mathrm{M}], \mathrm{BrO}_{3}[0.0553 \mathrm{M}]$, Petridish (id) $=9.1 \mathrm{~cm}$, thickness of solution $=0.1 \mathrm{~cm}$. Temp $=30^{\circ} \mathrm{C}$. (a) $=$ after 30 seconds (b) after 90 seconds (c) after 150 seconds.

Immediately after this fast pattern the system bifurcate to a stable red color for about one minute. After this break, the system shows concentric circular waves nucleation at some point in the petridish and moves outward with definite velocity. The single nucleation containing about 14 to 17 concentric wave fronts as shown in Figure 2.

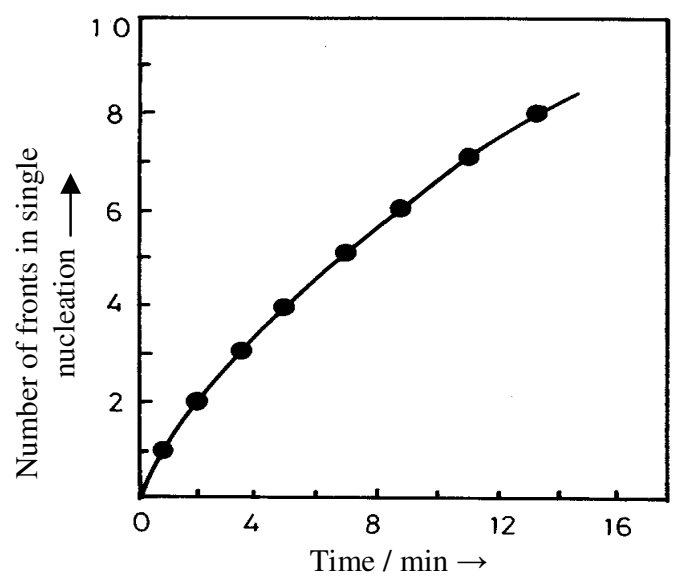

Figure 2. Number of concentric front nucleated against time. Concentration and conditions are same as in Figure 1.

The front velocity subsequently decreases with time i.e. velocity of second front is less than first, the velocity of third front is less than second front and so on. A typical photograph is shown in Figure 3. Several others nucleation's also occurs simultaneously and follows the same pattern. These nucleation processes continue to occur up to 10 to 15 minutes. When waves of two different nucleated fronts at different frequencies colloids each other, the high frequency front inhilates low frequency front. Further, this high frequency fronts colloids with other 
nucleated wave fronts and inhilates. Finally waves merges into a few concentric big ring and becomes stationary for 5 to 8 minutes, subsequently outer ring disappears first and gradually all rings disappears. These phenomena occur up to about 25-30 minutes from the beginning of the reaction. After that color of the phenomena turns to red. The first fast appearing and disappearing waves are appears to be due to oxidation and bromination of ethylacetoacetate corresponding to high frequency oscillations and second pattern are due to low frequency oscillations ${ }^{18}$. Again after 60 minutes from the beginning red color of the system turns blue which are remain stable for two hours. Then system shows red (liquid) nucleation on blue background of the system. These nucleations are not homogeneous throughout the petridish, which moves slowly in irregular manner. The process continues up to three hours. The concentration of red color increases with time and finally solid nucleation starts on red background. It I thought that this process is very significant in tree like pattern formation. Since there are new products formed and concentration of these products fluctuates in fascinating order. These fluctuations of concentration of products determine the shape, size and morphology of patterns.

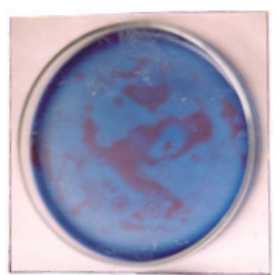

a

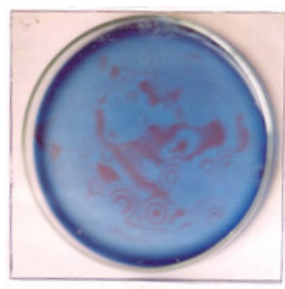

b

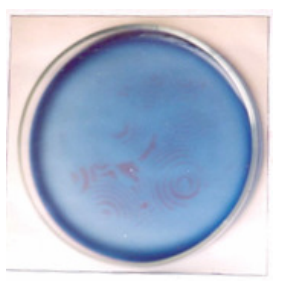

$\mathrm{c}$

Figure 3. Photographs of concentric wave pattern and nucleation. Concentrations and conditions are same as in Figure 1. (a) after 4 min (b) after 6 minutes (c) after 12 min.

After this break, systems show small nucleation at few places in the petridish and grow slowly into the form of trees / bushes type pattern both in height and breadths. A typical growth velocity is shown in Figure 4.

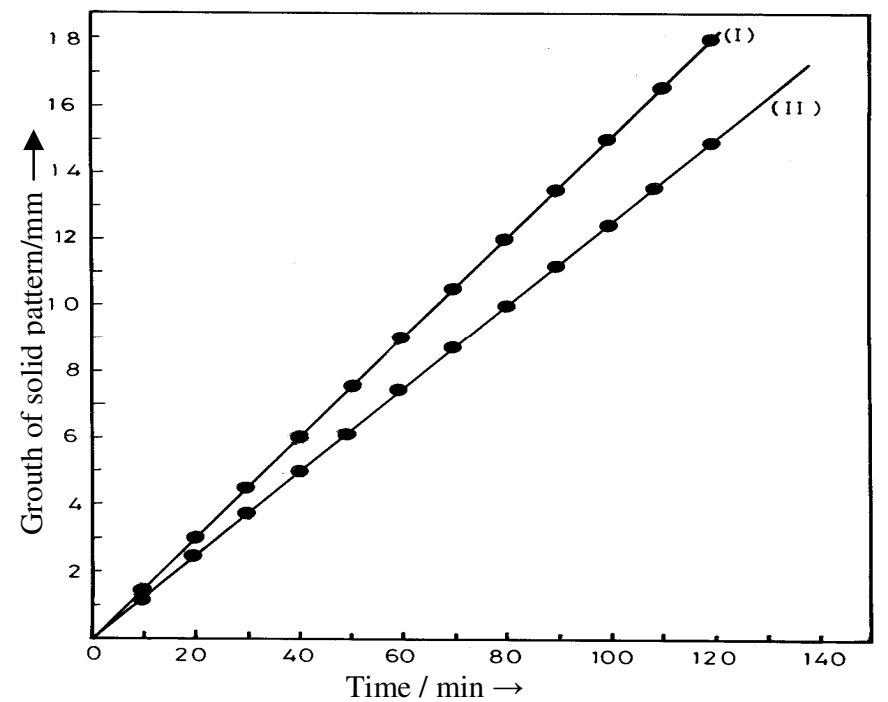

Figure 4. Growth of trees / bushes pattern against time. Concentration and conditions are same as in Figure 1. 1 = vertical growth, II = horizontal growth . 
The system takes 3 to 4 hours to produce fully grown trees / bushes like pattern. A typical photograph taken by a zoom lens camera is shown in Figure 5. The shape and nature of these trees / bushes like pattern changes on varying the initial concentration and condition of reactants. The color of the fully-grown pattern varies from yellow to red depending upon the initial composition of reactants and reaction temperature. On increasing the concentration of succinic acid pattern formed early but height and length decreases. This pattern remains stable up to 100 hours and then slowly decomposes. On keeping this pattern in a desiccator, it can be preserved for a long time. This means the pattern material is hygroscopic in nature. Atmospheric moisture destroys the structure.

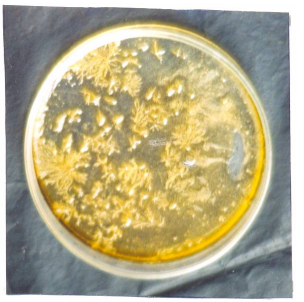

a

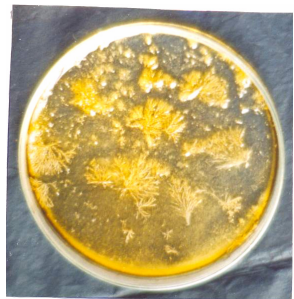

b

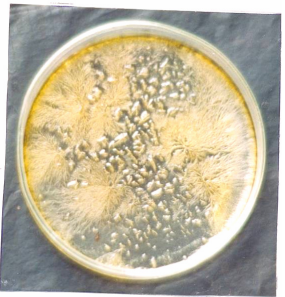

c

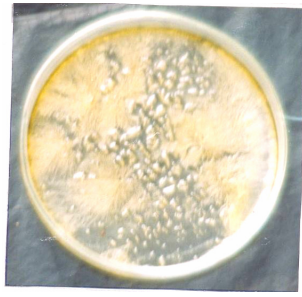

d

Figure 5. Photographs of trees / bushes patterns.

Concentration and conditions are same as in Figure 1, except a = Succinic Acid [0.1016 M], b = Succinic Acid [0.1125 M], c = Succinic Acid [0.1380 M], and d = Succinic Acid [0.1380 M], after $100 \mathrm{~h}$.

It was thought that pattern might be due to crystallisation of succinic acid or cerric ammonium sulphate. Therefore, the pattern material was purified by column chromatographic technique by taking methanol as solvent. Further, the pattern material was re-crystallized in methonol. The microphotographs of crystallized pattern material taken by Electronic microscope is shown in Figure 6(a) \& 6(b), which shows several nodes and branching in the form of long thin wires bunched together as appears in the structure. The microphotographs of succinic acid and cerric ammonium sulphate are shown in Figure 6(c) \& 6(d) respectively. The microphotograph of cerric ammonium sulphate shows small particles of yellow color material is uniformly distributed over the plate. Whereas microphotograph of succinic acid shows thick crystals of small size uniformly distributed over the plate. It is evident from the microphotographs of crystallized pattern material that the pattern material is entirely different product formed by periodic precipitation of two reaction products. It is further confirmed by Xray diffraction pattern of crystallized pattern material and that of succinic acid. X-ray diffraction pattern of pattern material and succinic acid are shown in figure 7(a) and 7(b) respectively, which the pattern material is crystalline in nature entirely different than succinic acid.

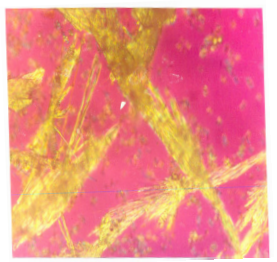

$400 X(a)$

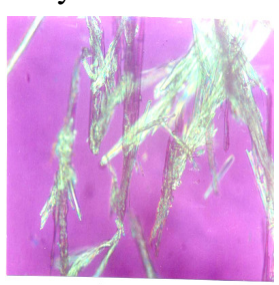

400X (b)

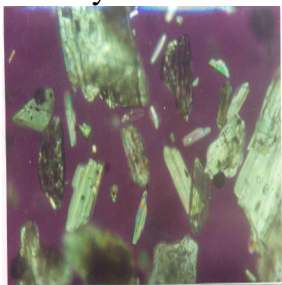

$400 X(c)$

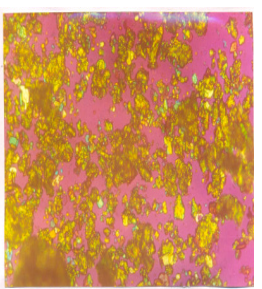

$400 X(d)$

Figure 6. Microphotograph of trees / bushes like pattern material along with microphotographs of succinic acid and ceric ammonium sulphate. (a) \& (b) = pattern material, $(\mathrm{c})=$ succinic acid,$(\mathrm{d})=$ ceric ammonium sulphate. 

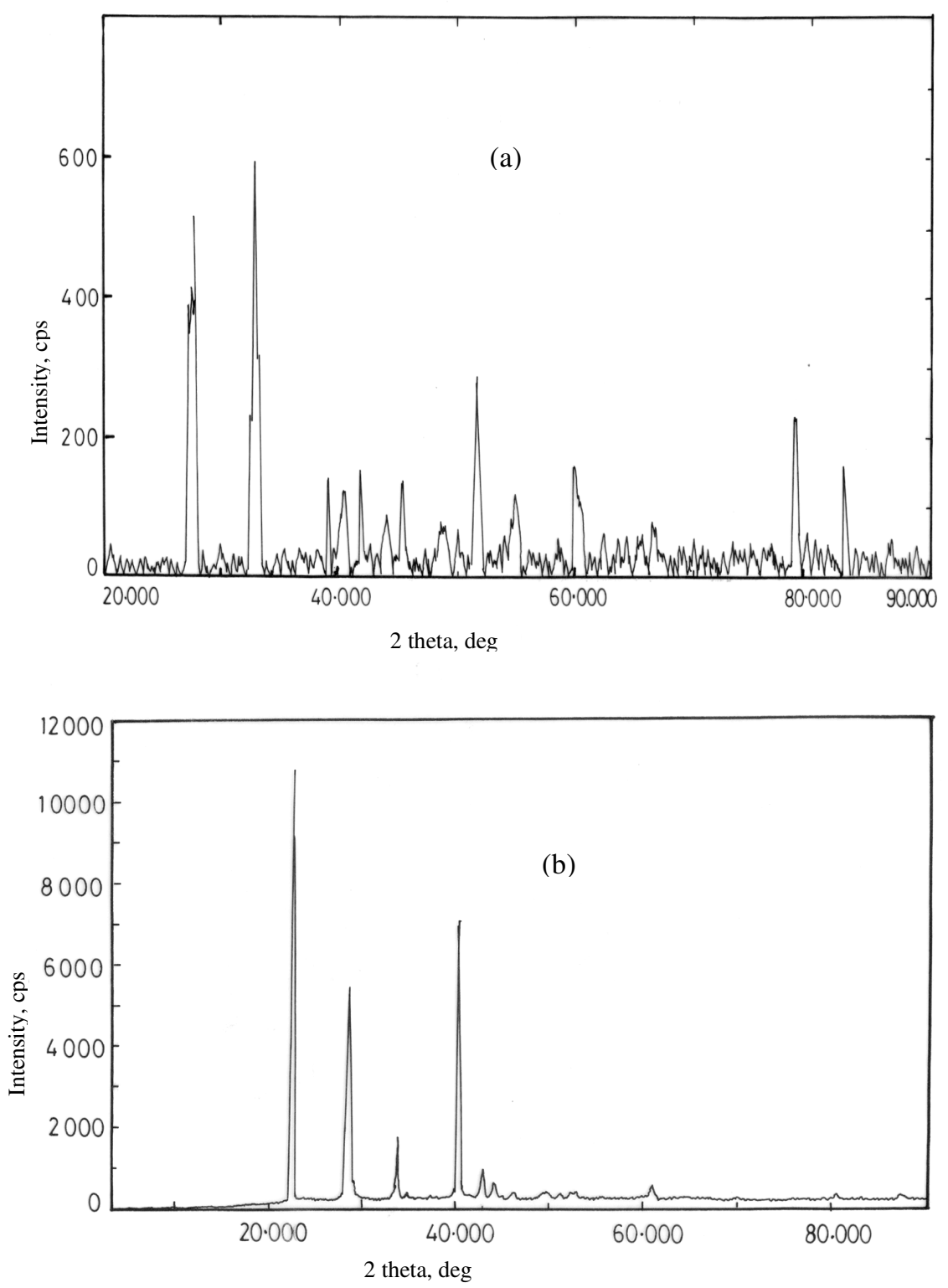

Figure 7. X-ray powder spectrum of trees / bushes pattern material along with X-ray powder spectrum of succinic. (a) Pattern material (b) Succinic acid.

\section{Mechanism of reactions}

Shelter and Shefford ${ }^{18}$ have reported that oxidation and bromination of ethylacetoacetate by bromate produces dibromoethylacetoacetate, hydroxy ethylacetoacetate and formyl acetone. 
It appears that the fast appearing / disappearing patterns and concentric ring pattern are due to oxidation / bromination of ethylacetoacetate. Further, four molecules of hydroxyl-ethylacetoacetate reacts with $\mathrm{Ce}\left(\mathrm{NH}_{4}\right)_{4}\left(\mathrm{SO}_{4}\right)_{4}$ in aqueous acidic medium and forms a $\mathrm{Ce}^{\mathrm{IV}}$ (hydroxyethylacetoacetate) ${ }_{4}$ which appears as clody material before the start of solid pattern nucleation. Simultaneously succinic acid decarboxylate and forms propionic acid. The oxidation of propionic acid in presence of cerium ion in acidic medium forms formyl acetic acid which undergo autooxidation to form malonic acid and finally dibromomalonic acid. Further, $\mathrm{Ce}^{\mathrm{IV}}$ (hydroxyethylacetoacetate) ${ }_{4}$ combines periodically with four $\left(-\mathrm{CH}_{2}-\right)$ or $\left(-\mathrm{CBr}_{2}-\right)$ or both unit of decarboxylated malonic acid/dibromomalonic acid to form trees / bushes like pattern (IV) or (V) or (VI). It is evident from the structure (IV), (V) and (VI) that it manifest the growth of pattern in all directions, as a reaction Scheme 1.

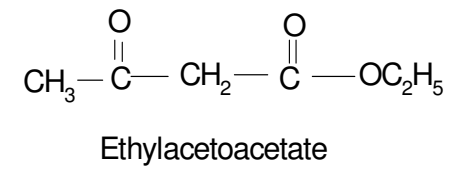

$$
\text { (i)oxidation (ii)bromination/Bro }{ }_{3}^{-}
$$<smiles>CCOC(=O)C(Br)(Br)C(=O)OCC</smiles>

Dibromoethylacetoacetate<smiles>CCOC(=O)C(O)C(C)=O</smiles>

(1)

$\mathrm{Ce}^{\mathrm{iv}}$ (hydroxyethylethylacetoacetate) ${ }_{4}$ 


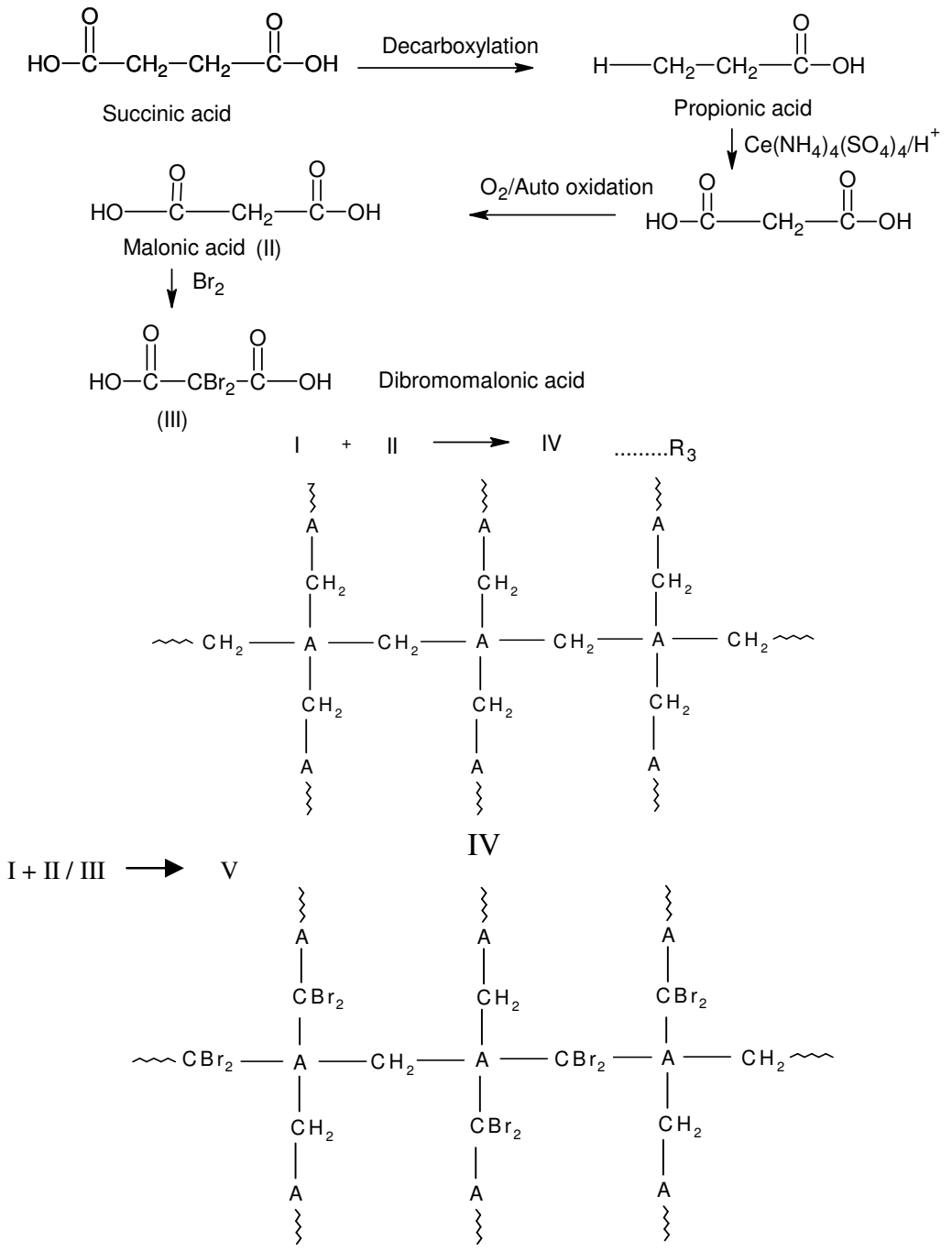

Where, $\quad \mathrm{A}=\mathrm{Ce}^{\mathrm{IV}}\left(\mathrm{hyd}_{(\mathrm{V})} \text { 'ethylacetoacetate }\right)_{4}$ $\mathbf{I}+\mathrm{III} \rightarrow \mathrm{VI}$

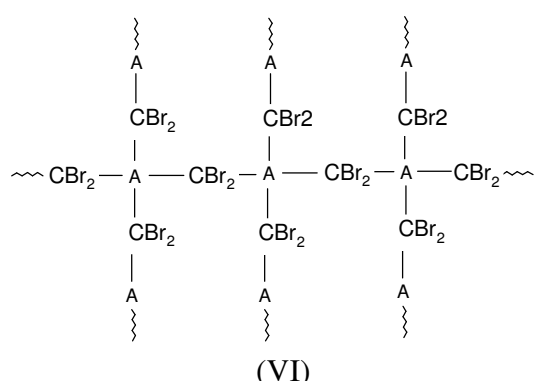

Scheme 1. 
The structure IV, V and VI of the pattern material is supported by the FT-IR spectrum as shown in Figure 8. This shows the presence of several bands ${ }^{19-23}$ below $1700 \mathrm{~cm}^{-1}$. A careful study of the spectrum shows the presence of cyclic structure as ring breathing band at $1598 \mathrm{~cm}^{-1}, 1542 \mathrm{~cm}^{-1}$ and $1510 \mathrm{~cm}^{-1}$ are present, along with $\mathrm{C}-\mathrm{H}$ deformation bands in di / tri substituted cyclic system at $885 \mathrm{~cm}^{-1}$ and $849 \mathrm{~cm}^{-1}$. The spectra shows the presence of metal - oxygen band at $575 \mathrm{~cm}^{-1}$ and the presence of $\mathrm{C}-\mathrm{O}$ stretching in metallic ester due to $1069 \mathrm{~cm}^{-1}$ and $1286 \mathrm{~cm}^{-1}$ vibrational band. Carbonyl $(\mathrm{C}=\mathrm{O})$ stretching band at $1700 \mathrm{~cm}^{-1}$ and bending vibration band at $1325 \mathrm{~cm}^{-1}$, are also present. Further, -C-O-C- stretching vibrational band at $1005 \mathrm{~cm}^{-1}, \mathrm{C}=\mathrm{C}$ stretching vibrational band at $1616 \mathrm{~cm}^{-1}, \quad \mathrm{C}-\mathrm{Br}$ stretching vibrational band at $614 \mathrm{~cm}^{-1}, \mathrm{OCH}_{2}-\mathrm{CH}_{3}$ strecthing vibrational band at $717 \mathrm{~cm}^{-1}$ alongwith $\mathrm{C}-\mathrm{H}$ deformation.

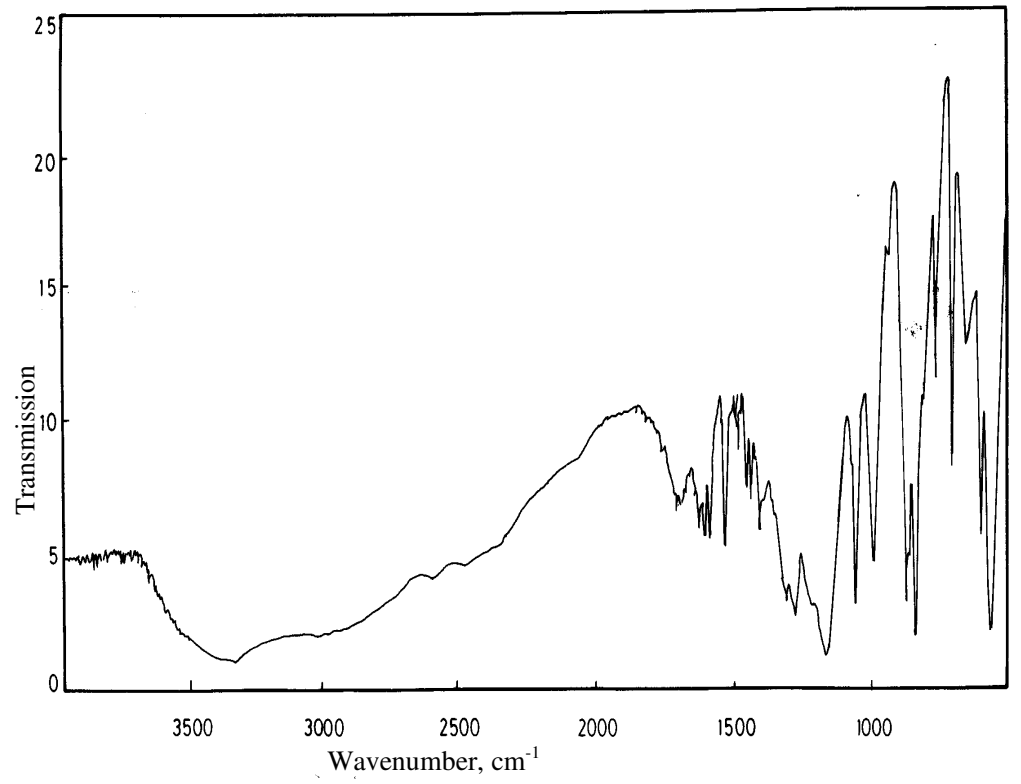

Figure 8. FT-IR spectrum of trees / bushes pattern material for conditions shown in Figure 1.

It appears that both the succinic acid and ethyl acetoacetate organic substances are important for trees / bushes pattern. This pattern does not occur with a single substrate as succinic acid or ethylacetoacetate. This pattern does not occur without ceric ammonium sulphate. Pattern is very sensitive to temperature, the time required for pattern formation decreases with increasing temperature. On increasing the thickness of the solution in petridish time required for tree / bush pattern increases. Pattern does not formed on closing the petridish, however pattern nucleates soon after removing the transparent cover of the petridish. If the pattern is half grown and we cover the petridish, no affect observes on growth. This shows that dehydration equilibrium is very important and is favours the pattern formation.

\section{Effect of oxygen on trees / bushes pattern}

It appears that oxygen play an important role in the last pattern formation, which is evident by the following experiments, on closing the petridish with transparent cover last pattern does not appear even for a long time. If we remove the cover of petridish pattern nucleates soon. To test the effect of oxygen on trees / bushes pattern, bromate from the reaction mixture was removed. It was found that in absence of bromate weak patterns appears, but growth of structures are very 
weak and small shown in Figure 9(a) \& 9(b) and depends on concentration, temperature and acidity. The microphotograph of pattern material in absence of bromate is shown in Figure 10. The formation of weak structure in absence of $\mathrm{BrO}_{3}$ can be explained on the basis of Scheme I. Oxidation of ethylacetoacetate into hydroxyethyacetoacetate in absence of bromate is very slow and formation of dibromalonic acid is not possible. Therefore, a limited concentration of hydroxyethylacetoacetate is available for formation of $\mathrm{Ce}^{\mathrm{IV}}$ (hydroxyethylacetoacetate) 4 . $\left.\left[\mathrm{Ce}^{\mathrm{IV}} \text { (hydroxyethylacetoacetate) }\right)_{4}\right]$ combines with four (-CH2-) unit of decarboxylated malonic acid which is available in limited concentration to form only structure IV. Therefore, pattern structure formed is small, thin, unbranched and spread uniformly throughout the petridish.

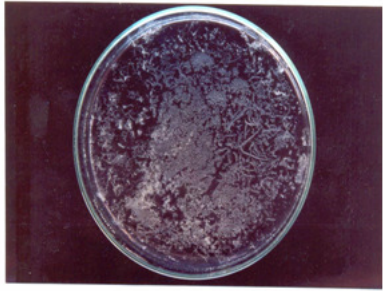

a

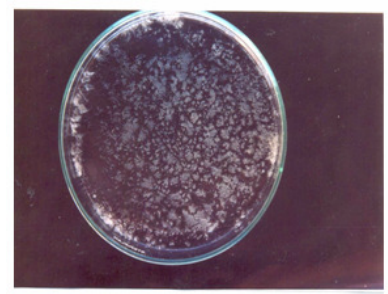

b

Figure 9. Photographs of trees / bushes like pattern in absence of bromate and ferroin, (a) Succinic Acid, [0.128 M], Ce ${ }^{+4},\left[0.022 \mathrm{M}\right.$ ], Ethylacetoacetate, [0.120 M], $\mathrm{H}_{2} \mathrm{SO}_{4}[2.5 \mathrm{M}]$, (b) Succinic Acid, [0.128 M], Ce ${ }^{+4},[0.022 \mathrm{M}]$, Ethylacetoacetate, [0.120 M], $\mathrm{H}_{2} \mathrm{SO}_{4}[1 \mathrm{M}]$, $i d=9.1 \mathrm{~cm}$, thickenss of solution $=0.1 \mathrm{~cm}$, Temperature $=30^{\circ} \mathrm{C}$.

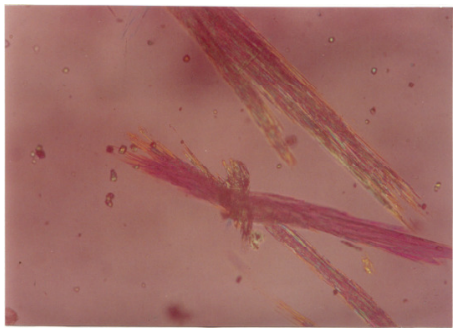

Figure 10. Microphotographs of trees and bushes like pattern without bromate and ferroin concentrations and conditions are same as in Figure 9.

\section{Effect of solvent on trees / bushes pattern}

The pattern material IV, V and VI are solvolized in polar solvents such as methanol and water. On dissolving the pattern materials into methanol,it solvolyzed as structure VII, VIII and IX. On evaporating the solvent molecules for 2 to 3 hours they re-aggalomerises and forms similar pattern. Similar behaviour was observed in water except time of agglomerisation which is 7 to 8 hours.

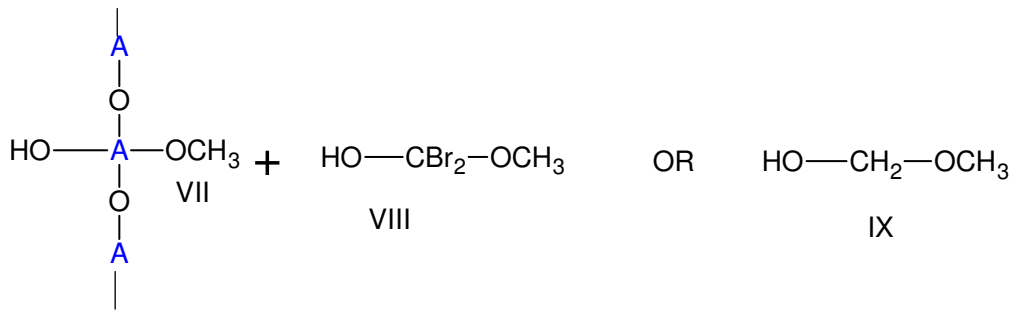




\section{References}

1. Hanna A, Saul and Showalter K, J Am Chem Soc., 1982, 104, 3838-3844; Zhabotinsky A M, Camp R N, Epstein I R and Lisman J E, Neurosci J., 2006, 26, 7337-7447.

2. Gribschaw T A, Showalter K, Banvilla D L and Epstein I R, J Phys Chem., 1981, 85, 2152.

3. Winfree A. T, Science, 1974, 175, 634; Vanag V K and Epstein I R, Patterns of Nanodroplets: The BZ-AOT Microemulsion System, in Self-Organized Morphology in Nanostructured Materials, Al-Shamery K and Parisi J, Ed., Springer, Berlin, 2008, pp. 89-113.

4. $\quad$ Keener J P and Tyson J J, Physica D, 1986, 21, 307.

5. Rastogi R P and Yadava K D S, Nature Physical Science, 1972, 240(97), 19-20.

6. Jahnke W and Winfree A T, J Chem Educ., 1991, 68, 320.

7. Sanders L M, The Physics of Structure Formation, Eds., Guttinger W and Gangelmayr G, Springer-Verlag, Berlin,1987, 257.

8. Kessler D A, Koplik J and Evine H L , Adv Phys., 1988, 37, 255.

9. Srivastava P K, Majhi S S, Rastogi R P and Hanazaki I, Chemistry Letter, 1998, 1251.

9. Daccord G and Lenormand R, Nature, 1987, 325, 41.

11. Palson E and Cox E C, Proc Natl Acad Sci., USA, 1996, 93, 1151.

12. Srivastava P K, Mori Y and Hanazaki I, J Phys Chem., 1991, 95, 1636; Srivastava P K and Majhi S S, J Indian Chem Soc., 2003, 80, 559- 561.

13. Henisch H. K., Crystals in Gels and Liesegang Ring, Cambridge University Press, New York, 1988, Vanag VK, Epstein IR, CHAOS 17(3): Art. No. 037110 SEP 2007

14. Das I., Mishra S. S., Agrawal N.R. and Gupta S. K, J Indian Chem Soc., 2003, 80, 351-360.

15. Michael Barnsley F., Dietmar Saupe, The Science of Fractual Images, Heinz-Otto Peitgen, Ditemar Saupe editors, Springer-Verlag, 1988, pp. 220.

16. Benoit Mandelbrat, The Fractal Geometry of Nature, W. H. Freeman and Co., San Francisco. 1983, Yang L, Dolnik M, Zhabotinsky A. M, and Epstein I R, Chaos, 2006, 16, 037114-1-9.

17. Dietmar Saupe, A Unified approach to fractal curves and plants, The Science of Fractal Images - Springer-Verlag, 1988, pp. 274.

18. Shelter F and. Shefford J G, Int J Chem Kinet., 1982, 14, 815.

19. Bellamy L. J., The Infrared spectrum of complex molecules, Volume I, $3^{\text {rd }}$ Ed., Chapman and Hall, London, 1975.

20. The systematic Identification of organic compounds, Editors Shriner, R. L., Hermann C.K.F, Morrill T C, Curtin D Y and Fuson R C, JohnWiley \& Sons, Inc. New York, 1998.

21. Herman A, Szymanski, Interpreted Infrared spectra, Volume I, Plenum Press, New York, 1964.

22. Tables of Spatial Data for structure determination of organic compounds, Editors, Erno Pretsch P.D, Seibl J, Simon W, Clerc T, translated by Biemann K, SpringerVerlag Berlin Heidelbrg, 1983.

22. Vogel A.I, A Textbook of Quantitative Inorganic Analysis, ELBS and Longmans Chapter X, 1968. 


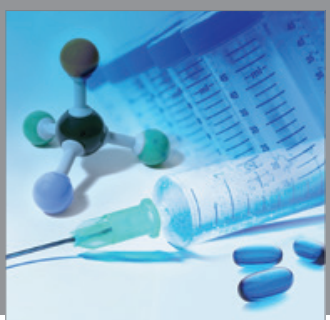

International Journal of

Medicinal Chemistry

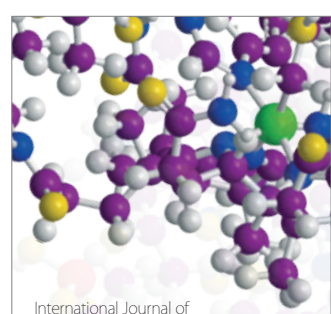

Carbohydrate Chemistry

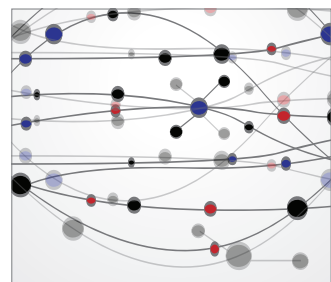

The Scientific World Journal
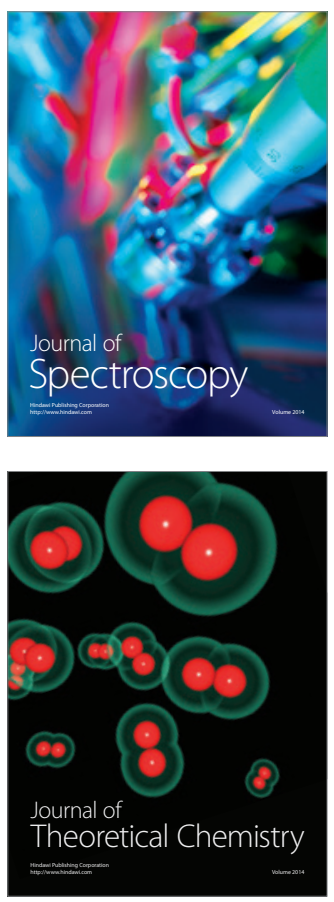
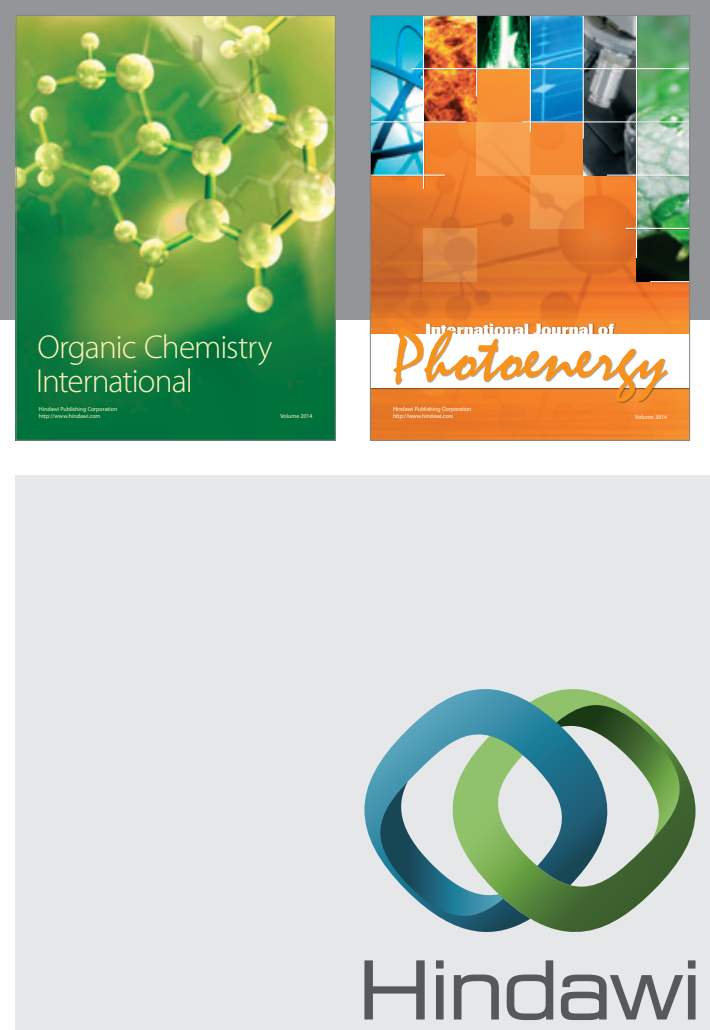

Submit your manuscripts at

http://www.hindawi.com
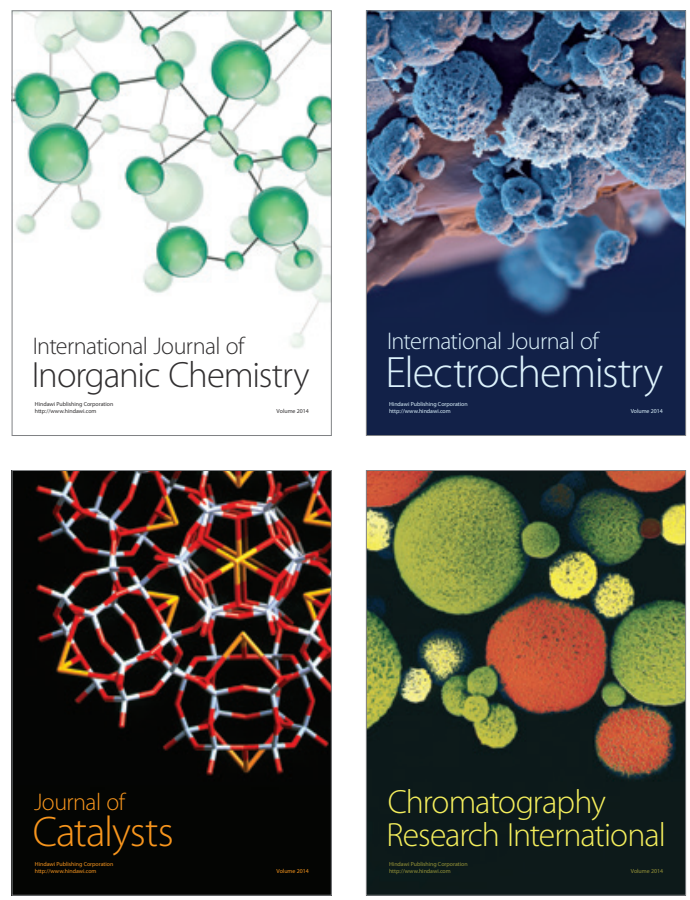
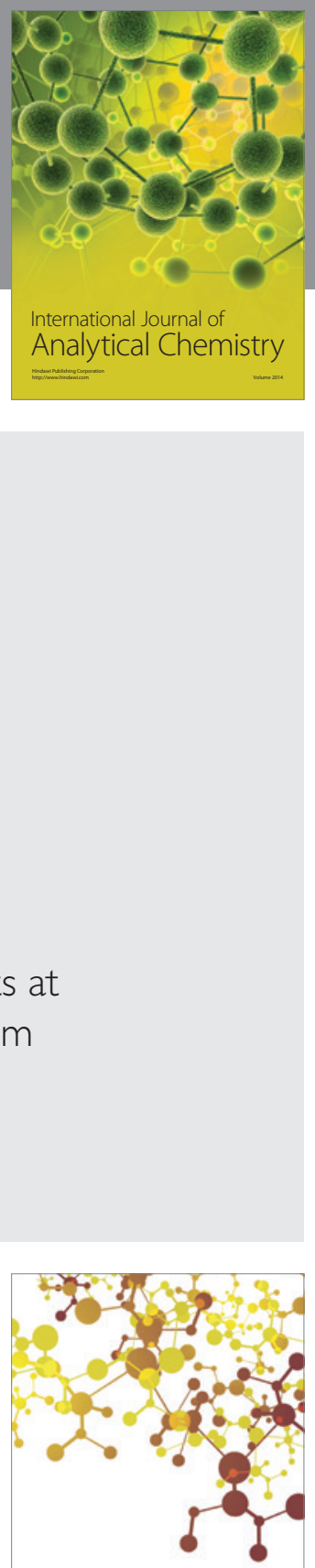

Journal of

Applied Chemistry
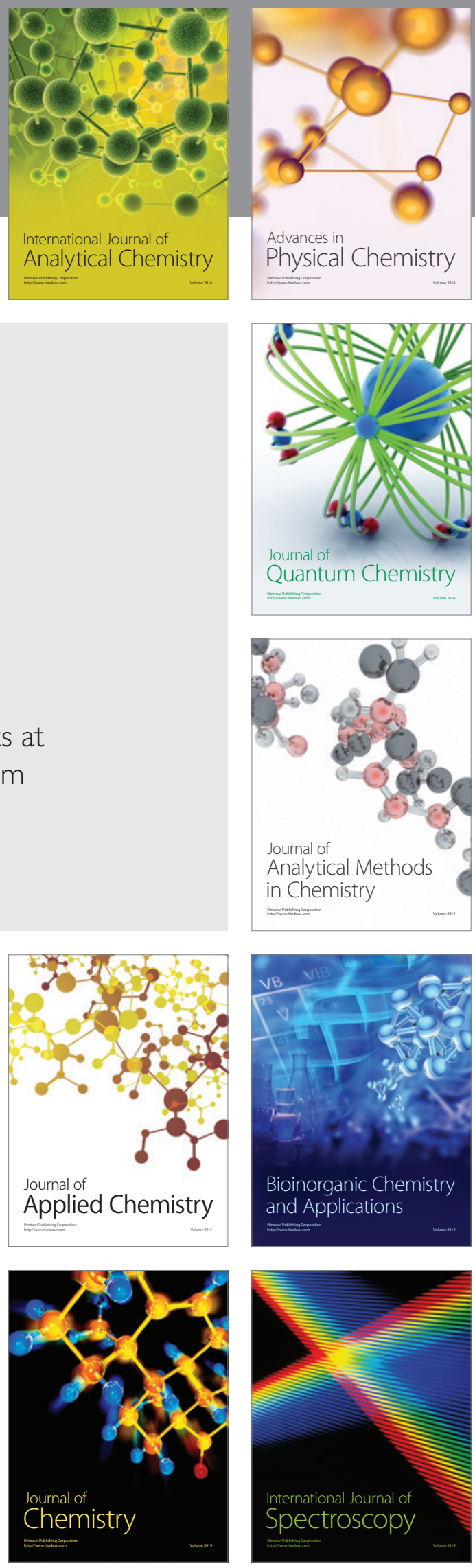